\title{
$M P Z$ mutation in an early-onset Charcot-Marie-Tooth disease type 1B family by genome-wide linkage analysis
}

\author{
BYUNG-OK CHOI $^{1 *}$, SANG-BEOM KIM ${ }^{4 *}$, SUMERA KANWAL ${ }^{5}$, YOUNG SE HYUN ${ }^{5}$, SUN WHA PARK ${ }^{5}$, \\ HEASOO KOO ${ }^{2}$, JEONG HYUN YOO ${ }^{3}$, JAE WON HYUN ${ }^{1}$, KEE DUK PARK ${ }^{1}$, \\ KYOUNG-GYU CHOI ${ }^{1}$ and KI WHA CHUNG ${ }^{5}$
}

\author{
Departments of ${ }^{1}$ Neurology, ${ }^{2}$ Pathology and ${ }^{3}$ Radiology, Ewha Womans University School of Medicine, Yangcheon-ku, \\ Seoul 158-710; ${ }^{4}$ Department of Neurology, Kyung Hee University, School of Medicine, Seoul; \\ ${ }^{5}$ Department of Biological Science and Research Center for Biotechnology, Kongju \\ National University, Gongju, Chungnam 314-701, Republic of Korea
}

Received January 31, 2011; Accepted March 24, 2011

DOI: $10.3892 / \mathrm{ijmm} .2011 .678$

\begin{abstract}
Charcot-Marie-Tooth disease (CMT) is a clinically and genetically heterogeneous peripheral neuropathy. The objective of this study was to find the causative mutation(s) in a demyelinating autosomal dominant CMT family. A high density SNP-based genome-wide linkage scan was performed, and causative mutations were determined by sequencing of candidate genes in the linkage disequilibrium region. Linkage analysis mapped the underlying gene to a region on chromosome 1q22-q23 with a maximum two-point LOD score of 2.036. Sequencing analysis revealed a novel c.243C $>\mathrm{G}$ (His81GIn) mutation in the $M P Z$ gene, which encodes the major integral membrane protein of the peripheral nerve system. $M P Z$ is well known as a CMT-causative gene with wide phenotypic spectrum. The clinical symptoms were more similar to those of patients with the His81Arg than patients with the His81Tyr mutation. The novel mutation completely co-segregated with affected members, and was not found in controls. Therefore, we suggest that the identified mutation in $M P Z$ is the underlying cause of CMT in the family. In addition, this study demonstrated that the clinical phenotypes may be variable with different mutations at the same site in the $M P Z$ gene.
\end{abstract}

\section{Introduction}

Hereditary motor and sensory neuropathy (HMSN), also called Charcot-Marie-Tooth disease (CMT), is a genetically

Correspondence to: Dr Ki Wha Chung, Department of Biological Science and Research Center for Biotechnology, Kongju National University, 182 Sinkwan-dong, Gongju, Chungnam 314-701, Republic of Korea

E-mail: kwchung@kongju.ac.kr

*Contributed equally

Key words: Charcot-Marie-Tooth disease, genome-wide scan, linkage analysis, $M P Z$, Korean and clinically heterogeneous disorder of the peripheral nervous system with a predominance of approximately 1 in 2500 people. CMT patients usually exhibit clinical symptoms of muscle weakness, sensory loss and foot deformities. CMT is classically divided into two types, the demyelinating type (CMT1) and the axonal type (CMT2), based on electrophysiological criteria. The demyelinating type has reduced median motor nerve conduction velocities (NCVs) $(<38 \mathrm{~m} / \mathrm{sec})$, whereas the axonal type has slightly reduced or normal NCVs $(>38 \mathrm{~m} / \mathrm{sec})$ $(1,2)$. A major reason for demyelination is glial dysfunction, which is responsible for malformation or destruction of the myelin sheath $(3,4)$. Demyelinating peripheral neuropathies usually ultimately lead to impaired function and degeneration of axons $(5,6)$.

The most frequent autosomal dominant demyelinating subtype is the CMT1A genomic disorder (MIM\# 118220), which is relevant with recurrent or non-recurrent rearrangements of chromosome $17 \mathrm{p} 12$ including the PMP22 gene (MIM\# 601097) (7-12). The CMT1B (MIM\# 118200) is the second most frequent autosomal dominant demyelinating subtype, and is relevant with MPZ (P0) gene (MIM\# 159440) mutations (13). However, $M P Z$ mutations are also associated with other phenotypes, including severe Dejerine-Sottas syndrome (DSS, MIM\# 145900) and axonal CMT, such as CMT2I (MIM\# 607677) and CMT2J (MIM\# 607736). These variable clinical phenotypes are usually associated with specific $M P Z$ mutations, namely different phenotypes by different mutation sites. Moreover, some $M P Z$ mutations have shown considerable clinical heterogeneity in the affected individuals not only among families, but also within the same family $(14,15)$.

To date, more than 50 genes or loci have been reported to be associated with CMT or related diseases at the Inherited Peripheral Neuropathies Mutation Database (IPNMD) (http:// www.molgen.ua.ac.be/CMTMutations/Mutations/) and in other reports. Genotype-phenotype correlations are occasionally less strict in CMT patients, and CMT displays clinically wide phenotypic heterogeneity according to causative mutation sites within a same gene. These factors make it difficult to 
predict specific genetic defects from several dozen causative genes. Therefore, narrowing chromosomal co-segregation region by the genome-wide linkage scan and subsequent sequencing analysis of a restricted number of candidate genes may be a more effective route rather than direct screening of all the CMT causative genes.

Two different mutations at the 81st amino acid site (His81) in the MPZ gene have been reported from CMT patients with different phenotypes: His81Arg in early-onset severe CMT1 $(16,17)$ and His81Tyr in late-onset mild CMT2 (18). In the present study, we identified another novel MPZ His81 mutation (His81Gln) in a Korean early-onset autosomal dominant demyelinating CMT family. For identification of the causative mutation, a high density SNP-based genome-wide linkage study and sequencing for the candidate genes were performed.

\section{Material and methods}

Subjects. This study included a total of 23 members ( 7 affected and 16 unaffected individuals) of a Korean early-onset autosomal dominant CMT1 family (family ID: FC133) (Fig. 1A). The paternity was confirmed by genotyping of 15 microsatellite markers using a PowerPlex 16 kit (Promega, Madison, WI). In addition, this study included 120 healthy controls who had no clinical features of neuromuscular disorder. Total DNA was extracted from the whole peripheral blood samples using a QIAamp DNA Blood mini kit (Qiagen, Hilden, Germany). All participants in this study provided written informed consent, and the study was approved by the Ethics Committee of Ewha Womans University, Mokdong Hospital.

Genome-wide linkage studies. A high density SNP-based genome-wide linkage scan was performed on 16 familial members by applying the Infinium II HumanLinkage-12 Panel (Illumina, San Diego, CA), to identify the linkage disequilibrium region. The chip included 6,090 SNP markers that are uniformly distributed on every chromosome with an average gap of $441 \mathrm{~kb}$ (approximately $0.58 \mathrm{cM}$ interval). Genotyping data were scanned by the Illumina BeadStation 500G array scanner, and analyzed with the Merlin-1.1.2 software using the autosomal dominant parametric model. SNP positions were obtained from the UCSC hg18 at the UCSC Genome Browser (http://genome.ucsc.edu/) or Build 36.1 at the NCBI website (http://blast.ncbi.nlm.nih.gov/).

Mutation screening. Sequencing analysis of all coding exons and flanking intronic sequences was performed on several candidate genes in the linkage disequilibrium region (1q22q23), and in several other CMT genes. We also examined the $1.4 \mathrm{Mb}$ duplication/deletion on 17.12 by the method of Choi et al (11). The primer sequences and the PCR conditions are available upon request. Sequencing reactions were performed using a BigDye Terminator v3.1 Cycle Sequencing kit, and sequences were then determined on an automatic genetic analyzer ABI 3100 (Applied Biosystems, Foster City, CA). Sequence variations were identified by the SeqScape (v.2.1, Applied Biosystems) and Chromas software (v.2.33, Technelysium, Australia). We confirmed the sequence variations by analyzing both strands of DNA. Multiple alignments of amino acid sequences among different species were performed with the ClustalX v.1.83 program.

Clinical assessment. Patients were evaluated by obtaining a detailed history including motor and sensory impairments, deep tendon reflexes, and muscle atrophy. Muscle strengths of flexor and extensor muscles were assessed manually using the standard medical research council (MRC) scale. In order to determine the physical disability we used two scales, the functional disability scale (FDS) (19) and the CMT neuropathy score (CMTNS) (20). Disease severity was determined for each patient using a nine-point FDS, which was based on the following criteria: 0 , normal; 1 , normal but with cramps and fatigability; 2 , an inability to run; 3 , walking difficulty but still possible to walk unaided; 4 , walking with a cane; 5 , walking with crutches; 6 , walking with a walker; 7, wheelchair bound; and 8, bedridden. Additionally, patients were divided into mild (CMTNS $\leq 10$ ), moderate (CMTNS, $11-20$ ), and severe (CMTNS $\geq 21$ ) categories. Sensory impairments were assessed in terms of the level and severity of pain, temperature, vibration, and position, and pain and vibration senses were compared.

Electrophysiological examination. Nerve conduction studies were tested with a surface electrode in median, ulnar, peroneal, tibial, and sural nerves. Motor nerve conduction velocities (MNCVs) of the median and ulnar nerves were determined by stimulation at the elbow and wrist while recording compound muscle action potentials (CMAPs) over the abductor pollicis brevis and adductor digiti quinti, respectively. In the same manner, the MNCVs of peroneal and tibial nerves were determined by stimulating at the knee and ankle, while recording CMAPs over the extensor digitorum brevis and adductor hallucis, respectively. Sensory nerve conduction velocities (SNCVs) were obtained over a finger-wrist segment from the median and ulnar nerves by orthodromic scoring, and were also recorded for sural nerves.

MRI study. Among the 7 affected patients, MRI was performed on 5 male patients (III-18, 20, IV-13, 14 and 17). Two patients did not receive an MRI assessment because of being too young (IV-16, 3 years and IV-18, 5 years). MRI was performed on the lumbar spine and lower legs in a supine position using a Siemens $1.5 \mathrm{~T}$ vision system (Siemens, Erlangen, Germany) equipped with a phase-array multicoil. Lower leg imaging was carried out in the axial [field of view (FOV) 24-32 cm, slice thickness $10 \mathrm{~mm}$, and slice gap 0.5-1.0 mm] and coronal planes (FOV 38-40 cm, slice thickness $4-5 \mathrm{~mm}$, slice gap $0.5-1.0 \mathrm{~mm}$ ). The following protocol was used for the 5 patients: T1-weighted spin-echo (SE) (TR/TE 570-650/1420, 512 matrixes), T2-weighted SE (TR/TE 2800-4000/96-99, 512 matrixes), and fat-suppressed T2-weighted SE (TR/TE 3090-4900/85-99, 512 matrixes) in both planes.

Histopathological study. The pathological examination of the proband (III-20) included light and electron microscopic analyses of the distal sural nerve. A fragment of peripheral nerve fiber was received in a fresh state. Formalin fixed sections with hematoxylin-eosin, modified Masson's trichrome, Luxol fast blue and Bodian stains were examined. 
A

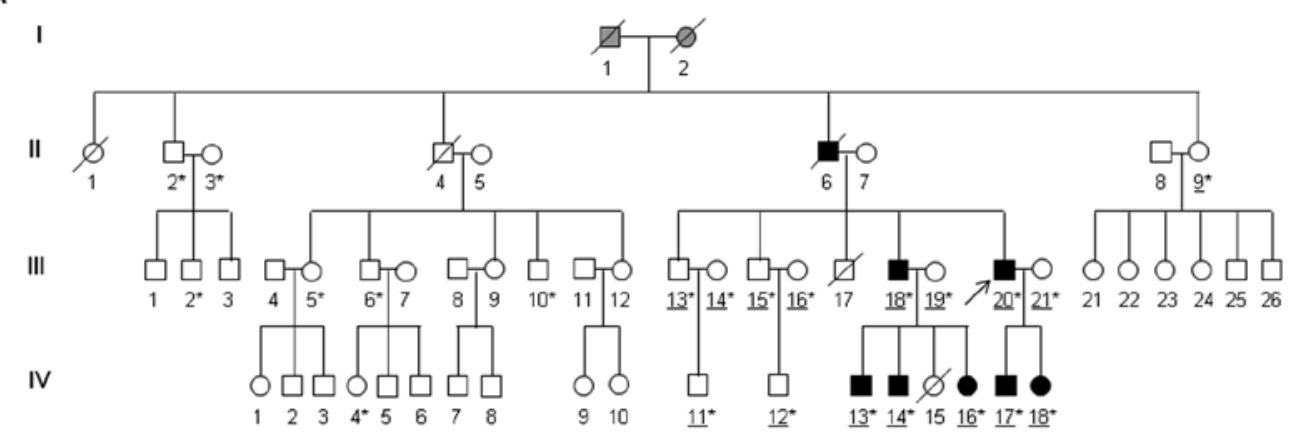

B
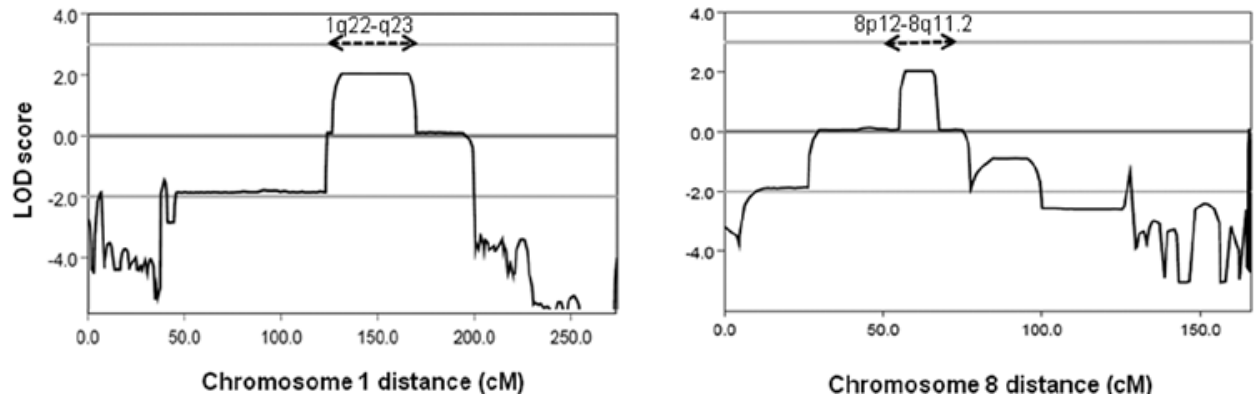

Figure 1. Pedigree of family FC133 and genome-wide SNP linkage analysis. (A) Pedigree of family FC133. *Individuals whose DNA was used for the mutation screening. Among them, underlined members are used for the genome-wide linkage analysis. Black, open, and grey symbols indicate affected, unaffected, and uncertain members, respectively. Arrow ( $(7)$ indicates proband. (B) Two-point LOD scores of chromosome 1 and 8 . A whole genome linkage scan was performed using a SNP chip, and LOD scores were obtained in the autosomal dominant model. High LOD scores were shown in the 1q22-q23 and 8p12-8q11.2 regions.

For the electron microscopic observations, the specimens were fixed in $2 \%$ glutaraldehyde in $0.025 \mathrm{M}$ cacodylate buffer

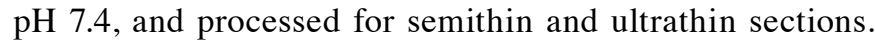
Semithin sections stained with toluidine blue were prepared for evaluation using light microscopy. Ultrathin cut samples (60-65 nm) were contrasted with uranyl acetate and lead citrate for an ultrastructural study (H-7650, Hitachi, Japan). The density of the myelinated fibers (MFs), the axonal diameter and myelin thickness, and the g-ratio of MFs (axon diameter/total fiber diameter) were assessed directly from the toluidine blue-stained semithin transverse sections of the sural nerve using a computer-assisted image analyzer (analySIS ${ }^{\circledR}$, Soft Imaging System, GmbH, Germany). The numbers of MFs with thick myelin or folding, thin myelinated axons, onion bulbs surrounding MFs, and regenerative clusters or axon sprouts (consisting of two or more adjacent myelinated axons surrounded by flattened Schwann cell processes) were assessed with electron microscopy at a lower magnification (x3,000). Unmyelinated fiber density was assessed from electron microscopic photographs at x10,000 magnification, taken to randomly cover uranyl acetate-stained ultrathin transverse sections.

\section{Results}

Identification of a linkage disequilibrium region on 1q22-q23. The genome-wide SNP scan revealed two candidate regions of linkage disequilibrium co-segregating with affected members, chromosome 1q22-q23 spanning from approximately $130-168 \mathrm{cM}$ and $8 \mathrm{p} 12-8 \mathrm{q} 11.2$ spanning from approximately 55-65 cM (Fig. 1B). The maximum obtained LOD scores were
2.036 at the SNP markers rs954679-rs1935745 (1q22-q23), and 2.035 at rs1000236-rs185596 (8p12-8q11.2) in the autosomal dominant inheritance model (theta $=0.00$ ).

The 1q22-q23 linkage disequilibrium region included two genes relevant with CMT, MPZ and $L M N A$. For the 8p12-8q11.2 region, no CMT-causative gene was reported; however, NEFL (8p21.2) and GDAP1 (8q21.1) genes were located on some distant vicinity of the disequilibrium region. The linkage study excluded several important CMT loci: 1p36.2 (MFN2), 10q21.2 (EGR2), 17p12 (PMP22), 19q13.2 $(P R X)$ and $\mathrm{Xq13.1}(G J B 1)$.

Identification of a novel missense mutation in MPZ. From the screening of the candidate genes on the 1q22-q23 linkage disequilibrium region, a missense change in exon 3 of $M P Z$ on 1q23.3 was identified as a functionally significant variant. The mutation was a $\mathrm{C}>\mathrm{G}$ transversion at position 243 (c. $243 \mathrm{C}>\mathrm{G}$ ), which leads to substitution of a histidine residue with glutamine, His81Gln (Fig. 2A). This mutation completely co-segregated with all affected members in the pedigree FC133 (III-18, III-20, IV-13, IV-14, IV-16, IV-17 and IV-18). Sequencing analysis confirmed the absence of this mutation in unaffected members of the pedigree and in 240 ethnically matched healthy control chromosomes. The His 81 mutation site is located on the extracellular immunoglobulin-like domain of the MPZ protein, and is highly conserved among other species (Fig. 2B). The c.243C $>\mathrm{G}$ mutation has not been reported in the dbSNP database (http://www.ncbi.nlm.nih.gov/snp/) or the Inherited Peripheral Neuropathies Mutation Database (http:// www.molgen.ua.ac.be/CMTMutations/Mutations/). However, two different mutations have been reported at the same His81 


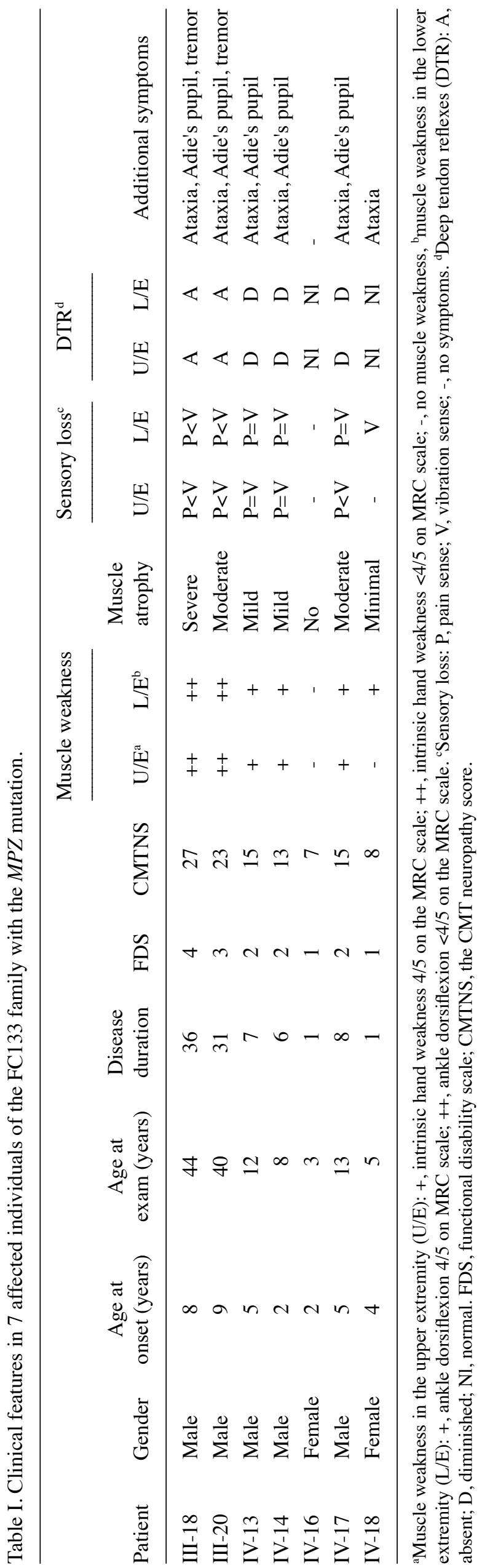

site: c. $241 \mathrm{C}>\mathrm{T}$ (His81Tyr) (18) and c.242A>G (His81Arg) $(16,17)$.

Sequencing analysis of $L M N A$ on 1q22, NEFL and GDAPI on chromosome 8 revealed no causative mutation. Although we excluded most CMT loci by the linkage study, mutational screening was performed for several other CMT genes. However, we could not find any causative mutation from the following genes: GJB1 (Cx32), EGR2, GARS, HSP22, HSP27, $P M P 22, P R X$ and YARS. In addition, 1.4 Mb duplication/ deletion on $17 \mathrm{p} 12$ was also excluded by genotyping of 6 microsatellites.

Clinical manifestations. The clinical features of the 7 patients ( 5 males, 2 females) are outlined in Table I. Muscle weakness and atrophy were first noted and predominated in the distal portions of the upper and lower limbs, and paresis in the distal regions varied from minimal to severe. Mean age at onset was $5.0 \pm 2.7$ years (range 2-9 years) and disease duration at the time of examination was $12.9 \pm 14.4$ years (range 1-36 years). Length-dependent sensory loss was found in 6 patients (86\%). Vibration sense was reduced to a greater extent than pain in 2 of the 5 patients, while in 3 patients it was reduced to a similar extent. Mean FDS was 2.1 \pm 1.1 (range 1-4 years) and CMTNS was $15.4 \pm 7.3$ (range 7-27 years). Two patients were in the severe category (CMTNS >21), and 5 of the 7 patients were in the mild to moderate category (CMTNS $\leq 20$ ). Pes cavus and ataxia were found in 6 patients $(86 \%)$, and postural hand tremor in 2 patients (29\%). The pupils of the proband were symmetric $(3 \mathrm{~mm})$ with absent light reflex and very slow near reaction and miosis in response to instillation of $0.1 \%$ pilocarpine. Pupil findings were identical to the patient's brother (III-18), son (IV-17), and cousins (IV-13 and -14). They did not have strabismus or ptosis. There was no evidence of either toxic habits or systemic disease, which could contribute to the observed neuropathy or papillary syndrome.

Electrophysiological findings. Neurophysiologic studies were carried out in all the affected individuals. MNCVs of median, ulnar, peroneal and tibial nerves, and SNCVs of median, ulnar and sural nerves are shown in Table II. The nerve conduction study demonstrated markedly reduced MNCVs, and the range of median MNCV was from 6.2-14.9 m/sec, and that of CMAP was from 0.1-3.8 mV. In patient (III-18), the amplitudes of evoked motor responses were markedly reduced, and we were unable to record amplitudes. All patients, except one with absent MNCVs, had at least one motor NCV $<38 \mathrm{~m} / \mathrm{sec}$. Sensory NCVs between finger and wrist were not recordable in all affected patients.

MRI findings. Among the 5 patients for whom MR images were obtained, all patients revealed grade I or II fatty infiltrations of both legs. Fatty infiltration was noted along the anterior and lateral compartment of both legs in a symmetric fashion. Fatty infiltration was greater in the III-18 and III-20 subjects (Fig. 3A and B) than in the IV-13, IV-14 and IV-17 (Fig. 3C-E), which indicates that fatty infiltration is related to disease duration. MRI features of the thigh were almost normal in all patients. These findings are similar to the reported features of demyelinating CMT1A neuropathy $(21,22)$. 
A

Control

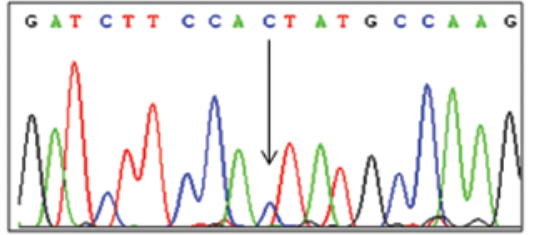

$\mathbf{B}$

H. sapiens

P. troglodytes

C. Iupus

B. taurus

M. musculus

R. norvegicus
Patient

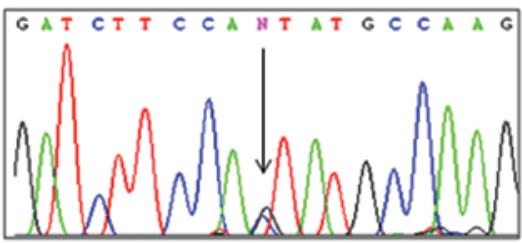

D I S TWR $Q P E G G R D A I S I$ FH YAKG $Q P Y I D E V G T F K E R I$

D I STWRYQPEGG RDA I S I H YAKG QPY I DEVG TF KERI

D LSFTWRYQPEGG RDAIS I FHYAKG QPY I DEVG TF KERI

D LSFTWRYQPEGGRDAISIFHYAKGQPYIDEVGTFKERI

D I STWRY QPEGG RDA I S I H YAKG QP Y I EVGAF KERI

DISFTWRYQPEGG RDAISIFHYAKGQPYIDEVG TF KERI

Figure 2. Identification of a missense mutation in the $M P Z$ gene. (A) Sequencing chromatograms of the c.243C $>\mathrm{G}$ (His $81 \mathrm{Gln}$ ) mutation in the $M P Z$ gene. The variant co-segregated with the affected family members. (B) Alignment of the amino acid sequences of the MPZ protein. High conservation of the His81 residue was revealed by alignment of the amino acid sequences of MPZ from different species (ClustalX program, v. 1.83).

Table II. Electrophysiological features in 7 affected individuals with MPZ mutation.

\begin{tabular}{|c|c|c|c|c|c|c|c|c|c|}
\hline \multirow[b]{2}{*}{ Patient } & \multirow[b]{2}{*}{ Age at exam } & \multirow[b]{2}{*}{ Side } & \multicolumn{4}{|c|}{ Motor nerve conduction study ${ }^{\mathrm{a}}$} & \multicolumn{3}{|c|}{ Sensory nerve conduction study } \\
\hline & & & Median & Ulnar & Peroneal & Tibial & Median & Ulnar & Sural \\
\hline \multirow[t]{2}{*}{ III-18 } & 44 & Right & NR & NR & NR & NR & NR & NR & NR \\
\hline & & Left & NR & NR & NR & NR & NR & NR & NR \\
\hline \multirow{2}{*}{ III-20 } & 40 & Right & $14.9(0.3)$ & $14.3(2.2)$ & NR & NR & NR & NR & NR \\
\hline & & Left & $12.2(0.1)$ & $13.5(3.3)$ & NR & $11.2(0.3)$ & NR & NR & NR \\
\hline \multirow[t]{2}{*}{ IV-13 } & 10 & Right & $11.0(3.7)$ & $7.4(5.5)$ & NR & $9.4(0.2)$ & NR & NR & NR \\
\hline & 12 & Left & $9.1(2.6)$ & $7.2(8.1)$ & NR & NR & NR & NR & NR \\
\hline \multirow[t]{2}{*}{ IV-14 } & 8 & Right & $11.5(3.8)$ & $8.0(3.0)$ & NR & NR & NR & NR & NR \\
\hline & & Left & $8.2(1.8)$ & $5.7(4.4)$ & NR & $6.5(0.2)$ & NR & NR & NR \\
\hline IV-16 & 3 & Right & $6.2(0.1)$ & $4.5(0.4)$ & NR & NR & NR & NR & $\mathrm{NR}$ \\
\hline \multirow[t]{2}{*}{ IV-17 } & 13 & Right & $12.3(3.3)$ & $10.2(4.5)$ & NR & NR & NR & NR & NR \\
\hline & & Left & $11.9(2.1)$ & $9.2(4.0)$ & NR & NR & NR & NR & NR \\
\hline IV-18 & 5 & Right & $9.2(1.7)$ & $8.9(3.0)$ & $8.3(0.5)$ & $4.9(0.1)$ & NR & NR & NR \\
\hline
\end{tabular}

${ }^{\mathrm{a}, \mathrm{b}} \mathrm{Amplitudes}$ of the evoked responses are given in parentheses [in $\mathrm{mV}$ for motor nerve conduction velocities (NCVs) and in $\mu \mathrm{V}$ for sensory $\mathrm{NCV}$ ] . Normal motor NCV values: median nerve $\geq 50.5 \mathrm{~m} / \mathrm{sec}$; ulnar nerve $\geq 51.1 \mathrm{~m} / \mathrm{sec}$; tibial nerve $\geq 41.1 \mathrm{~m} / \mathrm{sec}$; normal sensory NCV values: median nerve $\geq 39.3 \mathrm{~m} / \mathrm{sec}$; ulnar nerve $\geq 37.5 \mathrm{~m} / \mathrm{sec}$; sural nerve $\geq 32.1 \mathrm{~m} / \mathrm{sec}$. Normal amplitude values: motor median nerve $\geq 6 \mathrm{mV}$; ulnar nerve $\geq 8 \mathrm{mV}$; tibial nerve $\geq 6 \mathrm{mV}$; sensory median nerve $\geq 8.8 \mu \mathrm{V}$; sensory ulnar nerve $\geq 7.9 \mu \mathrm{V}$; sural nerve $\geq 6.0 \mu \mathrm{V}$. NR, not recordable.

Histopathological features. Semithin transverse sections showed severe loss of MFs of all calibers and the remaining MFs could be barely counted $412 / \mathrm{mm}^{2}$ (normal distal sural

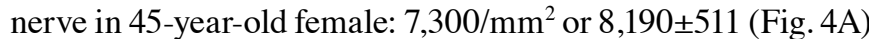
(23). Abnormally MFs with thick myelin (or folding) $(24.2 \%$, $\left.100 / \mathrm{mm}^{2}\right)$, thin myelinated axons $\left(6.1 \%, 25 / \mathrm{mm}^{2}\right)$, onion bulbs surrounding MFs $\left(42.4 \%, 175 / \mathrm{mm}^{2}\right)$, and regenerative clusters or axon sprouts $\left(12.1 \%, 50 / \mathrm{mm}^{2}\right)$ were observed. The histogram showed a unimodal distribution pattern with an axonal diameter $>8 \mu \mathrm{m}$ comprising $12.1 \%$ of MFs, and MFs $<3 \mu \mathrm{m}$ were absent (Fig. 4B). The range of the diameter and the average diameter of MFs were 3.4-11.0 and 5.7 $\mu \mathrm{m}$, respectively (normal distal sural nerve in 45-year-old female, 1.8-14.8 and 5.4 $\mu \mathrm{m}$ ). The MF\% area in this case was $1.1 \%$ (normal distal sural nerve of 45-year-old female, 26.9\%). The range of the g-ratio was 0.6-0.8 (normal distal sural nerve of 42-year-old male, 0.6-0.7) (23). A g-ratio $>0.7$ comprised $60.6 \%$ of MFs and none of the fibers showed a g-ratio $<0.4$. Electron microscopic examination revealed onion bulbs surrounding myelinated and unmyelinated fibers and axon sprouts (Fig. 4C), abnormally MFs with excessive myelin outfoldings or focally folded myelin exhibiting degenerative 

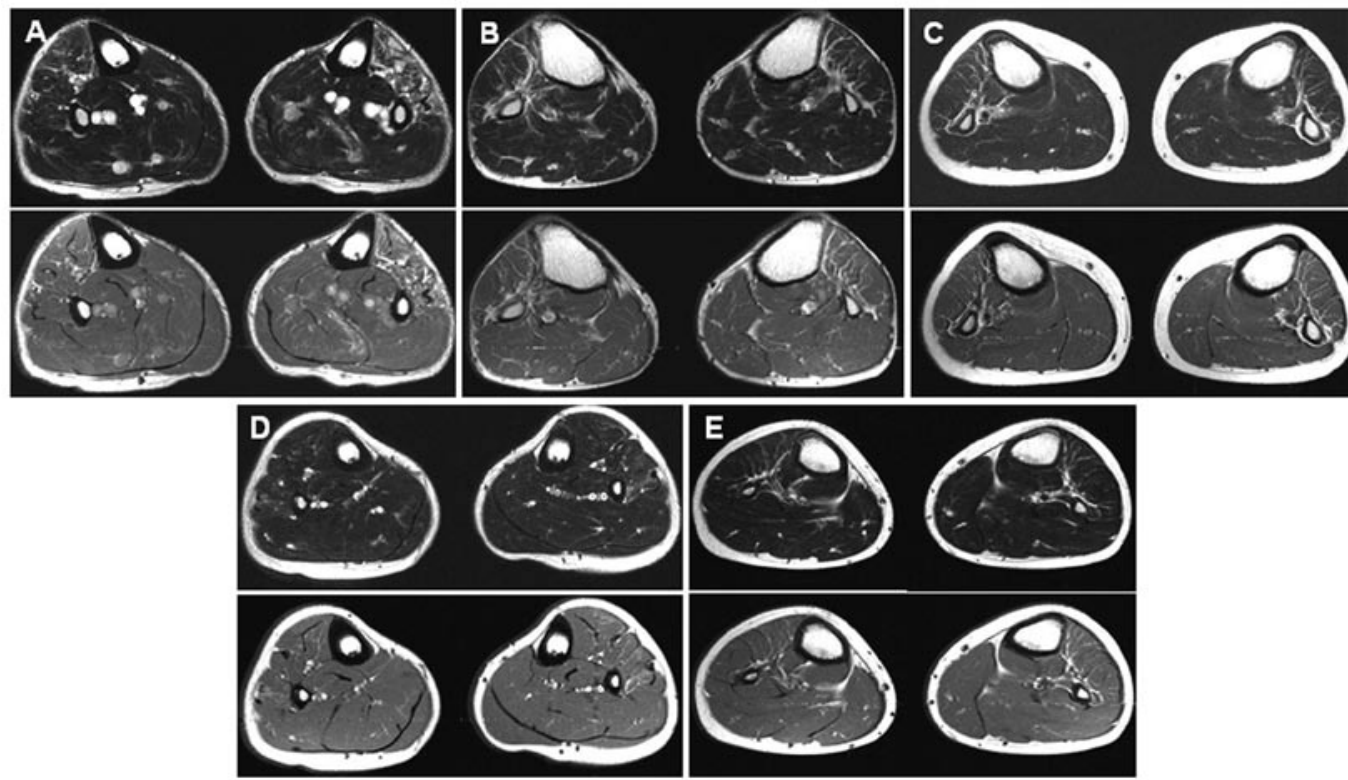

Figure 3. Disease duration (DD)-dependent fatty infiltration of low leg MR imagings in the patients with the $M P Z$ mutation. T1-weighted spin-echo (upper) and T2-weighted spin-echo (lower) images in (A) a 44-year-old male patient (III-18, DD, 36 years), (B) a 40-year-old male patient (III-20, DD, 31 years), (C) a 13-year-old male patient (IV-17, DD, 8 years), (D) a 12-year-old male patient (IV-13, DD, 7 years) and (E) an 8-year-old male patient (IV-14, DD, 6 years). Lower leg MRIs show grade I or II fatty infiltrations of both legs. Fatty infiltration is noted along the anterolateral compartment of both legs in a symmetric fashion. Greater fatty infiltration was observed in (A) and (B) the parents' generation than in (C-E) the children's generation. In addition, (A) also shows high signal intensity of the left anterolateral muscle group, suggestive of accompanying muscle edema.
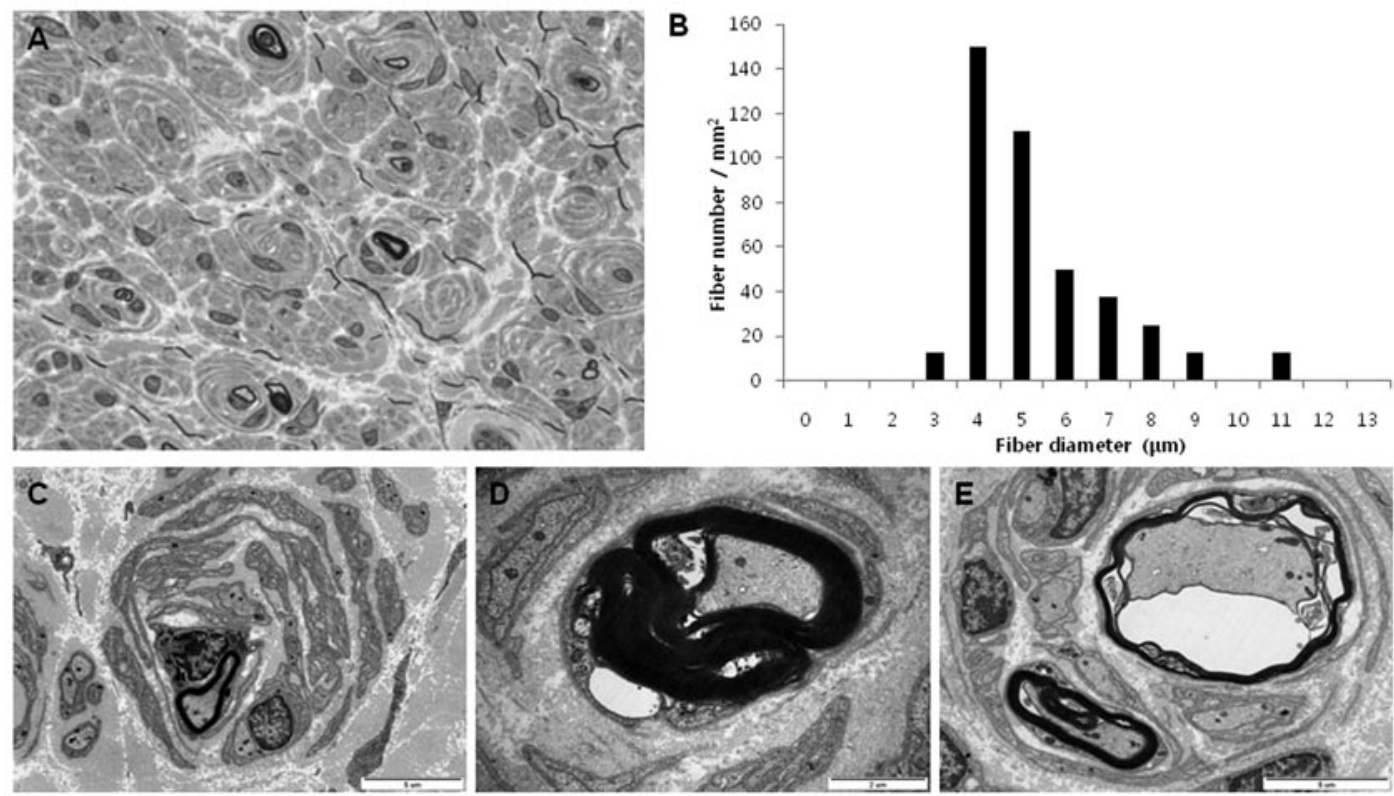

Figure 4. Histopathological features of distal sural nerve biopsy. (A) Light microscopic examination of semithin section. It reveals severe loss of myelinated fibers with onion bulbs surrounding myelinated and unmyelinated fibers, abnormally myelinated fibers with thick folded myelin, and thinly myelinated fibers. (B) Histogram of unimodal distribution pattern. The histogram shows an axonal diameter $>8 \mu \mathrm{m}$ comprising $12.1 \%$ of the myelinated fibers. Myelinated fibers $<3 \mu \mathrm{m}$ were absent. (C-E) Ultrastructural micrographs. They show onion bulb formation surrounding myelinated fiber (C), abnormally myelinated fiber with excessive myelin outfolding or focally folded myelin (D), and thinly myelinated fiber with uncompacted myelin (E). (A, toluidine blue stained semithin section, x1,000; C, ultrathin section, x8,000; D, ultrathin section, x20,000; E, ultrathin section, x10,000).

changes (Fig. 4D), uncompacted myelin, and thinly myelinated axons (Fig. 4E). Compared with MFs, the unmyelinated fibers were relatively well preserved and were counted as $65,763 /$ $\mathrm{mm}^{2}$ (control, $28,400 / \mathrm{mm}^{2}$ or $29,913 \pm 3,457$ ) (23). Endoneurial fibroblast proliferation and large amounts of collagen deposition have been well documented.

\section{Discussion}

We identified two linkage disequilibrium regions on chromosome 1q22-q23 and 8p12-8q11.2 in the FC133 autosomal dominant CMT1 family by the genome-wide SNP linkage study. Sequencing analysis revealed a novel c. $243 \mathrm{C}>\mathrm{G}$ 
(His81Gln) transversion mutation in the $M P Z$ gene, which encodes a major component of myelin of the peripheral nerve system. Our data support that the His81Gln mutation in the $M P Z$ is responsible for the autosomal dominant demyelinating CMT in family FC133 due to the following reasons: i) co-segregation of the mutation with affected members in the pedigree, ii) no detection of the same mutation in 240 ethnicity matched control chromosomes, iii) high conservation of amino acids at the mutation site among different species, iv), absence of alternative causative mutations in known CMT genes and v) close matching of the phenotype with the $M P Z$ mutations.

$M P Z$ encodes a $29 \mathrm{kDa}$ transmembrane glycoprotein, which is the major structural protein of peripheral myelin. Expression of $M P Z$ is restricted to Schwann cells, and plays a role in linking adjacent lamellae and thereby stabilizing the myelin assembly. MPZ protein belongs to the immunoglobulin supergene family, and contains three domains: an extracellular domain, a transmembrane domain and an intracellular C terminal domain $(24,25)$. After Bird et al mapped the autosomal dominant CMT1B locus on 1q22-q23 (26), Hayasaka et al identified the $M P Z$ mutations as the underlying cause of CMT1B (13). To date, more than a hundred MPZ mutations have been reported to be relevant with CMT. Mutations in $M P Z$ are shown to be responsible for a wide range of CMT phenotypes from mild to severe and from early-onset to lateonset (27-29). In particular, the marked phenotypic variations on the same $M P Z$ mutation suggest other putative modifying gene(s) $(14,15)$.

The His81Gln mutation locates on the extracellular immunoglobulin-like domain. The extracellular domain plays an important role in myelin compaction, and most CMT-causative mutations are localized within the extracellular domain. In particular, the His81 residue has been suggested to influence the adhesion interface between homotypic interactions (30). Although His81Gln has not been previously reported, two mutations have been reported in the His81 site. His81Arg has been described in early-onset CMT1 patients $(16,17)$. Notably, a Japanese female patient with the mutation exhibited severe CMT1 with bilateral trigeminal thickening and an abnormality of the auditory brain stem response (17). The mutation of His81Tyr has been identified to be concurrent with another heterozygous mutation, Val113Phe, in a late-onset CMT female with axonal neuropathy and pupillary light-near dissociation (18). The symptoms were less severe than for patients having His 81 Arg. In our case, the family FC133 showed the typical CMT1 phenotype similarly to the patients with His81Arg. The clinical features showed demyelinating CMT1 neuropathies in His81 Arg and His81Gln; however, His81Tyr displayed axonal CMT2 neuropathy. A sural nerve pathological finding with His81Tyr showed mainly axonal neuropathy with only a few onion bulbs; however, the present 40-year-old male revealed features of demyelinating neuropathy such as frequent onion bulb formations (42.4\%) as well as MFs with thick myelin or folding (24.2\%) and thin myelinated axons (6.1\%). In addition, on the MRI, the patients with the MPZ mutation showed grade I-II fatty infiltration along the anterolateral compartment of the lower leg, similarly to the reported feature of CMT1A neuropathy $(21,22)$. It is interesting that variable phenotypes were observed even among subjects with mutations at the same 
site of the MPZ (Table III). In a similar case, two Pro22 mutations in NEFL genes revealed different phenotypes: Pro22Ser in CMT2E and Pro22Thr in CMT1F (31).

According to the present study, the novel $M P Z$ mutation appears to be the causative mutation for the CMT disease in the CMT1 family. We also found that the clinical phenotypes may vary even among different mutations at the same site in the $M P Z$ gene.

\section{Acknowledgements}

This study was supported by the Mid-career Researcher Program through an NRF grant funded by the MEST (R012008-000-20604-0), the Korea Healthcare Technology R\&D Project, Ministry for Health, Welfare and Family Affairs (A090500), Republic of Korea.

\section{References}

1. Skre H: Genetic and clinical aspects of Charcot-Marie-Tooth's disease. Clin Genet 6: 98-118, 1974.

2. Bouche P, Gherardi R, Cathala HP, Lhermite F and Castaigne P: Peroneal muscular atrophy. Part I. Clinical and electrophysiological study. J Neurol Sci 61: 389-399, 1983.

3. Hanemann CO: Hereditary demyelinating neuropathies: from gene to disease. Neurogenetics 3: 53-57, 2001.

4. Shy ME, Balsamo J, Lilien J and Kamholz J: A molecular basis for hereditary motor and sensory neuropathy disorders. Curr Neurol Neurosci Rep 1: 77-88, 2001.

5. Martini R: The effect of myelinating Schwann cells on axons. Muscle Nerve 24: 456-466, 2001.

6. Young $P$ and Suter U: Disease mechanisms and potential therapeutic strategies in Charcot-Marie-Tooth disease. Brain Res Brain Res Rev 36: 213-221, 2001.

7. Lupski JR, Montes de Oca-Luna R, Slaugenhaupt S, Pentao L, Guzzetta V, Trask BJ, Saucedo-Cardenas O, Barker DF, Killian JM, Garcia CA, Chakravarti A and Patel PI: DNA duplication associated with Charcot-Marie-Tooth disease type 1A. Cell 66: 219-232, 1991.

8. Zhang F, Khajavi M, Connolly AM, Towne CF, Batish SD and Lupski JR: The DNA replication FoSTeS/MMBIR mechanism can generate genomic, genic and exonic complex rearrangements in humans. Nat Genet 41: 849-853, 2009.

9. Zhang F, Seeman P, Liu P, Weterman MA, Gonzaga-Jauregui C, Towne CF, Batish SD, De Vriendt E, De Jonghe P, Rautenstrauss B, Krause KH, Khajavi M, Posadka J, Vandenberghe A, Palau F, Van Maldergem L, Baas F, Timmerman V and Lupski JR: Mechanisms for nonrecurrent genomic rearrangements associated with CMT1A or HNPP: rare CNVs as a cause for missing heritability. Am J Hum Genet 86: 892-903, 2010.

10. Huang J, Wu X, Montenegro G, Price J, Wang G, Vance JM, Shy ME and Züchner S: Copy number variations are a rare cause of non-CMT1A Charcot-Marie-Tooth disease. J Neurol 257: 735-741, 2010.

11. Choi BO, Kim J, Lee KL, Yu JS, Hwang JH and Chung KW: Rapid diagnosis of CMT1A duplications and HNPP deletions by multiplex microsatellite PCR. Mol Cells 23: 39-48, 2007.

12. Weterman MA, van Ruissen F, de Wissel M, Bordewijk L, Samijn JP, van der Pol WL, Meggouh F and Baas F: Copy number variation upstream of PMP22 in Charcot-Marie-Tooth disease. Eur J Hum Genet 18: 421-428, 2010.

13. Hayasaka K, Himoro M, Sato W, Takada G, Uyemura K, Shimizu N, Bird TD, Conneally PM and Chance PF: CharcotMarie-Tooth neuropathy type 1B is associated with mutations of the myelin P0 gene. Nat Genet 5: 31-34, 1993.
14. Szabo A, Züchner S, Siska E, Mechler F and Molnar MJ: Marked phenotypic variation in a family with a new myelin protein zero mutation. Neuromuscul Disord 15: 760-763, 2005.

15. Mazzeo A, Muglia M, Rodolico C, Toscano A, Patitucci A, Quattrone A, Messina C and Vita G: Charcot-Marie-Tooth disease type 1B: marked phenotypic variation of the Ser78Leu mutation in five Italian families. Acta Neurol Scand 118: 328-332, 2008.

16. Sorour E, MacMillan J and Upadhyaya M: Novel mutation of the myelin P0 gene in a CMT1B family. Hum Mutat 9: 74-77, 1997.

17. Shizuka M, Ikeda Y, Watanabe M, Okamoto K, Shoji M, Ikegami T and Hayasaka K: A novel mutation of the myelin $\mathrm{P}(0)$ gene segregating Charcot-Marie-Tooth disease type 1B manifesting as trigeminal nerve thickening. J Neurol Neurosurg Psychiatry 67: 250-251, 1999.

18. Bienfait HM, Baas F, Gabreëls-Festen AA, Koelman JH, Langerhorst CT and de Visser M: Two amino-acid substitutions in the myelin protein zero gene of a case of Charcot-Marie-Tooth disease associated with light-near dissociation. Neuromuscul Disord 12: 281-285, 2002

19. Birouk N, LeGuern E, Maisonobe T, Rouger H, Gouider R, Tardieu S, Gugenheim M, Routon MC, Léger JM, Agid Y, Brice A and Bouche P: X-linked Charcot-Marie-Tooth disease with connexin 32 mutations: clinical and electrophysiologic study. Neurology 50: 1074-1082, 1998.

20. Shy ME, Blake J, Krajewski K, Fuerst DR, Laura M, Hahn AF, Li J, Lewis RA and Reilly M: Reliability and validity of the CMT neuropathy score as a measure of disability. Neurology 64: 1209-1214, 2005

21. Gallardo E, Garcia A, Combarros O and Berciano J: CharcotMarie-Tooth disease type 1A duplication: spectrum of clinical and magnetic resonance imaging features in leg and foot muscles. Brain 129: 426-437, 2006.

22. Chung KW, Suh BC, Shy ME, Cho SY, Yoo JH, Park SW, Moon H, Park KD, Choi KG, Kim S, Kim SB, Shim DS, Kim SM, Sunwoo IN and Choi BO: Different clinical and magnetic resonance imaging features between Charcot-Marie-Tooth disease type 1A and 2A. Neuromuscul Disord 18: 610-618, 2008.

23. Sobue G, Hashizume Y, Mukai E, Hirayama M, Mitsuma T and Takahashi A: X-Linked recessive bulbospinal neuronopathy: a clinicopathological study. Brain 112: 209-232, 1989.

24. Lemke $G$ and Axel R: Isolation and sequence of a cDNA encoding the major structural protein of peripheral myelin. Cell 40: 501-508, 1985.

25. Eichberg J: Myelin P0: new knowledge and new roles. Neurochem Res 27: 1331-1340, 2002.

26. Bird TD, Ott J and Giblett ER: Evidence for linkage of CharcotMarie-Tooth neuropathy to the Duffy locus on chromosome 1. Am J Hum Genet 34: 388-394, 1982.

27. Shy ME, Jáni A, Krajewski K, Grandis M, Lewis RA, Li J, Shy RR, Balsamo J, Lilien J, Garbern JY and Kamholz J: Phenotypic clustering in MPZ mutations. Brain 127: 371-384, 2004.

28. Zschüntzsch J, Dibaj P, Pilgram S, Kötting J, Gerding WM and Neusch C: Severe demyelinating hypertrophic polyneuropathy caused by a de novo frameshift mutation within the intracellular domain of myelin protein zero (MPZ/P0). J Neurol Sci 281: 113-115, 2009.

29. Lee YC, Lin KP, Chang MH, Liao YC, Tsai CP, Liao KK and Soong BW: Cellular characterization of $M P Z$ mutations presenting with diverse clinical phenotypes. J Neurol 257: 1661-1668, 2010.

30. Shapiro L, Doyle JP, Hensley P, Colman DR and Hendrickson WA: Crystal structure of the extracellular domain from P0, the major structural protein of peripheral nerve myelin. Neuron 17: 435-449, 1996.

31. Shin JS, Chung KW, Cho SY, Yun J, Hwang SJ, Kang SH, Cho EM, Kim SM and Choi BO: NEFL Pro22Arg mutation in CharcotMarie-Tooth disease type 1. J Hum Genet 53: 936-940, 2008. 